\title{
Photodegradation of organic compounds adsorbed in porous mineral layers: determination of quantum yields
}

\author{
Supporting Information
}

ANDREA CIANI*, KAI-UWE GOSS*, AND RENÉ P. SCHWARZENBACH

Swiss Federal Institute for Environmental Science and Technology (EAWAG), Postfach 611, CH 8600

Dübendorf, Switzerland and Swiss Federal Institute of Technology (ETH), Zürich, Switzerland

*corresponding author: phone ++41 4482354 68, e-mail: goss@eawag.ch, andrea.ciani@gmx.ch 


\section{Spectra of the lamps used}

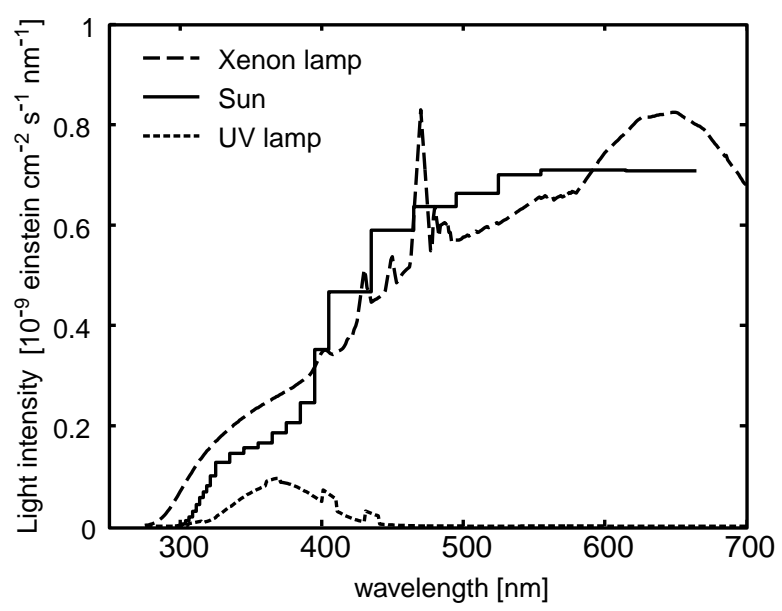

Figure S1. Spectra of the xenon and UV lamp (corrected with the pyrex glass transmittance) used in this work compared with the sunlight intensity from (1). The lamp spectra are determined with actinometry experiments and by scaling the spectra published by the manufacturer.

\section{Absorption and scattering coefficients for kaolinite}

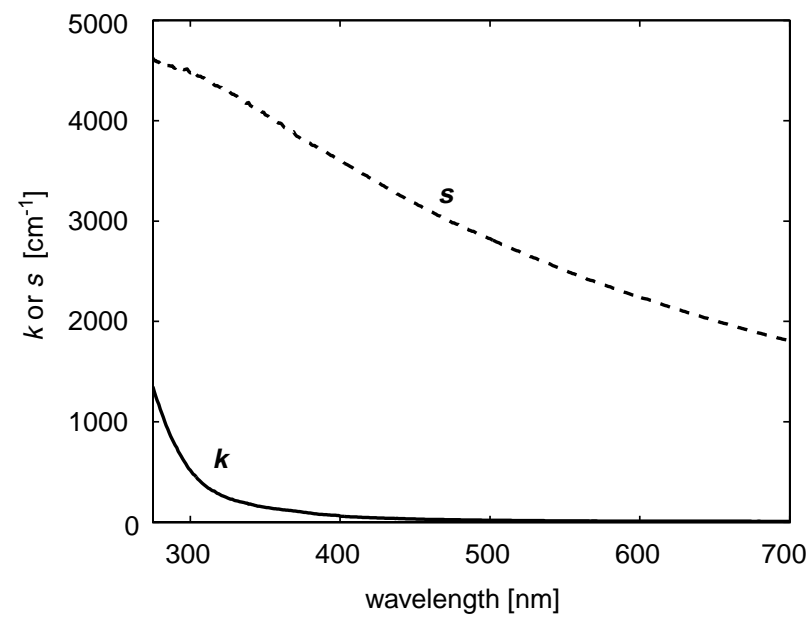

Figure S2. Light absorption and scattering coefficients for kaolinite (2). 


\section{Molar absorption spectra of PNA and trifluralin adsorbed on kaolinite}

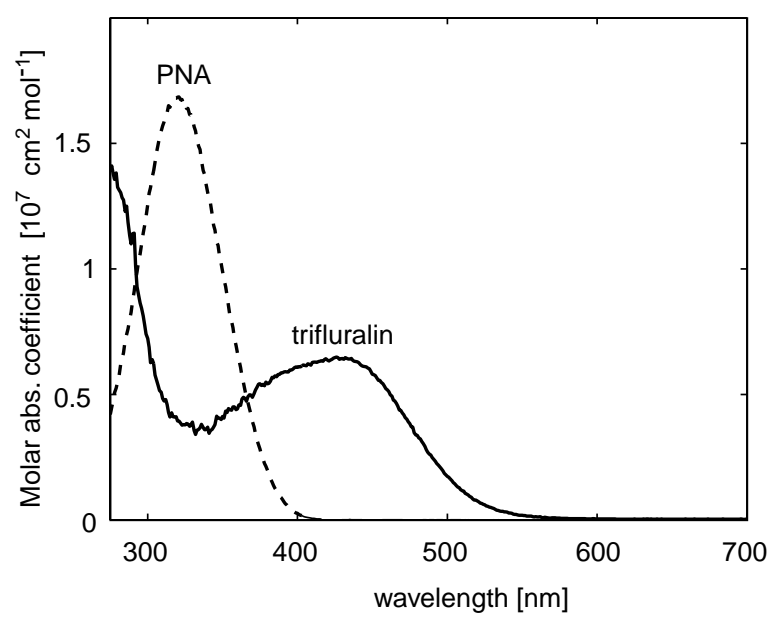

Figure S3. Molar absorption spectra of PNA and trifluralin adsorbed on kaolinite (3).

4. Details about method C: Spectroscopic Determination of the Quantum Yield. From the change of the diffuse reflectance during the irradiation of the porous layer, the reaction quantum yield can be derived with a method similar to that described by Gade \& Porada $(4,5)$, which was developed for reversible photoisomerization reactions in light scattering media. Gade \& Porada used the KubelkaMunk model for the description of the light intensity in the medium and assumed the compounds to be completely immobile in the porous layer. This assumption of no diffusion in the layer is reasonable for rapid photoreactions and only for the beginning phase of the experiment when the concentration gradient is small. In the following, the theory of these calculations is shortly described, with an adaptation to our case where we assume the photoreaction to be irreversible.

If a compound $i$ is irradiated and transformed into the product $p$, the reflectance and transmittance of a layer will change during irradiation, assuming that $i$ and $p$ have different molar absorption coefficients $\varepsilon_{i}(\lambda)$ and $\varepsilon_{p}(\lambda)$, respectively. The set of equations describing these phenomena is based on the Kubelka-Munk model (5):

$$
\begin{aligned}
& \frac{\partial C_{i}(t, \lambda, z)}{\partial t}=-\Phi_{i}(\lambda) 2 \ln (10) \varepsilon_{i}(\lambda)[I(t, \lambda, z)+J(t, \lambda, z)] \\
& \frac{\partial C_{p}(t, \lambda, z)}{\partial t}=-\frac{\partial C_{i}(t, \lambda, z)}{\partial t}
\end{aligned}
$$




$$
\begin{aligned}
& \frac{\partial I(t, \lambda, z)}{\partial z}=-q(t, \lambda, z) I(t, \lambda, z)+\mathrm{s} J(t, \lambda, z) \\
& \frac{\partial J(t, \lambda, z)}{\partial z}=q(t, \lambda, z) J(t, \lambda, z)-\mathrm{s} I(t, \lambda, z)
\end{aligned}
$$

where $q(t, \lambda, z) \equiv k+s+2 \ln (10)\left[\varepsilon_{i}(\lambda) C_{i}(t, \lambda, z)+\varepsilon_{p}(\lambda) C_{p}(t, \lambda, z)\right]$. Note that for the description of $\partial C_{i}(t, \lambda, z) / \partial t$ eq 10 cannot be used because in this case the concentration of $i$ is higher and the inner filter effect substantial. This set of equations is valid only for the case that one product $p$ is formed; in case that several products are formed eqs S1-S4 must be extended with the corresponding equations and quantum yield.

In order to solve eqs. S1-S4 for the quantum yield, various quantities must be known. The following quantities are assumed to be constant with time and space: $k, s, \varepsilon_{i}(\lambda), \varepsilon_{p}(\lambda)$ and $\Phi_{i}(\lambda)$. The molar absorption coefficient $\varepsilon_{i}(\lambda)$ can be determined with diffuse reflectance measurements of doped layers (3) before a reaction took place, whereas the molar absorption coefficient of the product $p, \varepsilon_{p}(\lambda)$, can be determined from the reflectance after complete reaction. There are four variables which are time and space dependent: the concentrations $C_{i}(t, \lambda, z)$ and $C_{p}(t, \lambda, z)$ and the light fluxes $I(t, \lambda, z)$ and $J(t, \lambda, z)$. The only parameters that are measurable are the ratios $R=J(t, \lambda, z=0) / I_{0}(\lambda)$ and $T=I(t, \lambda, z=d) / I_{0}(\lambda)$, that is, the reflectance and transmittance of the layer during the reaction (Figure 1).

The solution of eqs S1-S4 is tricky because during the irradiation of the layer a concentration profile of $i$ and $p$ builds up. Consequently the absorption coefficient $k_{\text {tot }}$ will be a function of time and depth in the layer. Under these circumstances the classical solution of the Kubelka-Munk equations ( 6 ) is not applicable anymore, because it assumes a constant light absorption coefficient, $k_{\text {tot }}$, with depth (that is, it assumes a homogeneous concentration of $i$ with depth).

One method consists in a Taylor series approximation for optically thick layers exhibiting socalled infinite reflectance, based on the work of Gade \& Porada $(4,5)$. The initial slope of the infinite reflectance curve versus irradiation time is used to calculate the quantum yield. If an optically thick layer is irradiated with a defined wavelength (denoted with the superscript ${ }^{\diamond}$ ) and if the molar absorption 
coefficients $\varepsilon_{i}^{\diamond}$ and $\varepsilon_{p}^{\diamond}$ are different, it is possible to calculate the quantum yield of the reaction with the initial slope of the graph of the infinite reflectance $R_{\infty, i p}^{\diamond}$ versus time $\left(\partial R_{\infty, i p}^{\diamond} / \partial t\right)$. The quantum yield can then be calculated with the following equation derived from Gade $\&$ Porada $(4,5)$ :

$$
\Phi_{i}^{\diamond} \approx\left[\frac{\partial R_{\infty, i p}^{\diamond}}{\partial t}\right] \frac{3 \varepsilon_{i}^{\diamond} C_{i}(t=0, \mathrm{z})\left(1-R_{\infty, i}^{\diamond}\right)}{4 I_{0}^{\diamond} s^{\diamond}\left(\varepsilon_{i}^{\diamond}-\varepsilon_{p}^{\diamond}\right)\left[f\left(R_{\infty, i}^{\diamond}\right)-f\left(R_{\infty}^{\diamond}\right)\right]^{2} R_{\infty, i}^{\diamond 2}}
$$

where $f\left(R_{\infty}\right) \equiv\left(1-R_{\infty}\right)^{2} /\left(2 R_{\infty}\right)$ is the so-called Kubelka-Munk function and the infinite reflectance of the pure medium and of the doped medium are given with $R_{\infty}^{\diamond}$ and $R_{\infty, i}^{\diamond}$, respectively. Alternatively, in case that $\varepsilon_{i}^{\diamond}$ and $\varepsilon_{p}^{\diamond}$ are similar (no reflectance change observed during irradiation), the quantum yield can be determined by evaluating the reflectance change with time at a wavelength different from that of the irradiation (denoted with superscript ${ }^{\bullet}$ ). The corresponding equation for the determination of the quantum yield is:

$$
\Phi_{i}^{\diamond} \approx\left[\frac{\partial R_{\infty, i p}^{\bullet}}{\partial t}\right] \frac{\varepsilon_{i}^{\bullet} C_{i}(t=0, \mathrm{z})\left[2 R_{\infty, i}^{\diamond}\left(1-R_{\infty, i}^{\bullet 2}\right)+R_{\infty, i}^{\bullet}\left(1-R_{\infty, i}^{\diamond 2}\right)\right]}{4 I_{0}^{\diamond} s^{\diamond}\left(\varepsilon_{i}^{\bullet}-\varepsilon_{p}^{\bullet}\right)\left[f\left(R_{\infty, i}^{\bullet}\right)-f\left(R_{\infty}^{\bullet}\right)\right]\left[f\left(R_{\infty, i}^{\diamond}\right)-f\left(R_{\infty}^{\diamond}\right)\right] R_{\infty, i}^{\bullet 2} R_{\infty, i}^{\diamond}\left(1+R_{\infty, i}^{\diamond}\right)}
$$

\section{Details about method D: Extended Spectroscopic Approach.}

The Kubelka-Munk model can describe the radiative transfer for media whose optical properties $(k$ and $s$ ) are homogeneous in the depth of the layer (6). For nonhomogeneous optical properties (e.g. there is more compound near the surface), the reflectance $R$, the transmittance $T$ as well as the light fluxes $I$ and $J$ have to be calculated numerically with eqs S3-S4 and by dividing the layer in $n$ thin slices of thickness $\Delta z$, whose $k$ and $s$ values are approximately constant (Figure S4). 


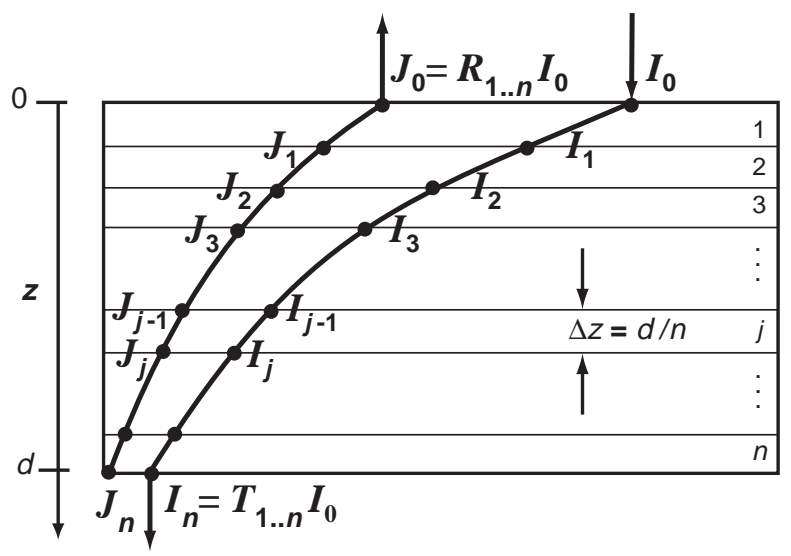

Figure S4. Calculation of $I$ and $J$ of a layer with inhomogeneous concentrations of $i$ and $p$.

The reflectance $R_{j . n}$ and transmittance $T_{j . . n}$ of the pile of slices from slice $j$ to $n$ can be calculated iteratively with following equations beginning from $j=n$ up to $j=1(7-10)$ :

$$
\begin{aligned}
& R_{j . . n}=R_{j}+\frac{T_{j}^{2} R_{j+1 . . n}}{1-R_{j} R_{j+1 . . n}} \\
& T_{j . . n}=\frac{T_{j} T_{j+1 . . n}}{1-R_{j} R_{j+1 . . n}}
\end{aligned}
$$

where $R_{j}$ and $T_{j}$ are the reflectance and transmittance of the single slice $j$ calculated with its average light absorption value $k_{\text {tot }}$ (proportional to the concentration $C_{i}(\mathrm{z}=j \cdot \Delta z)$ ) and its thickness $d=\Delta z$ according to following equations:

$$
\begin{aligned}
& R_{j}=\frac{\left(1-\beta^{2}\right)\left(\mathrm{e}^{\alpha d}-\mathrm{e}^{-\alpha d}\right)}{(1+\beta)^{2} \mathrm{e}^{\alpha d}-(1-\beta)^{2} \mathrm{e}^{-\alpha d}} \\
& T_{j}=\frac{4 \beta}{(1+\beta)^{2} \mathrm{e}^{\alpha d}-(1-\beta)^{2} \mathrm{e}^{-\alpha d}}
\end{aligned}
$$

The light intensity at a depth $z=j \cdot \Delta z$ in the layer, $I_{j}$ and $J_{j}$, can be calculated with (similar to $(7,9)$ ):

$$
I_{j}=\frac{I_{j-1} T_{j}}{1-R_{j} R_{j+1 . . n}}
$$




$$
J_{j}=I_{j} R_{j+1 . . n}
$$

By knowing $I_{j}$ and $J_{j}$ (eqs S11-S12) it is possible to solve eqs S1-S2 numerically. The disappearance of $i$ and production of $p$ in a small time interval $\Delta t$ can be calculated with the expressions:

$$
\begin{aligned}
& \Delta C_{i}(t+\Delta t, j \cdot \Delta z)=-\Phi_{i}^{\diamond} 2 \ln (10) \varepsilon_{i} C_{i}(t, j \cdot \Delta z)\left[I_{j}(t, j \cdot \Delta z)+J_{j}(t, j \cdot \Delta z)\right] \Delta t \\
& \Delta C_{p}(t+\Delta t, j \cdot \Delta z)=-\Delta C_{i}(t+\Delta t, j \cdot \Delta z)
\end{aligned}
$$

where $\mathrm{C}_{i}(t+\Delta t, j \cdot \Delta z)$ and $\mathrm{C}_{p}(t+\Delta t, j \cdot \Delta z)$ are the concentration of $i$ and $p$ at the time $t+\Delta t$ at a depth of $j \cdot \Delta z$ in the layer. For an improved calculation one can also implement the diffusion kinetics of $i$ and $p$ in the layer (with Cranck-Nicholson scheme). The numerical procedure consists of the following steps: (i) calculation of $I_{j}(t+\Delta t, j \cdot \Delta z)$ and $J_{j}(t+\Delta t, j \cdot \Delta z)$ with the concentration profiles of $i$ and $p$ at time $t$ (eqs S11 -S12); (ii) calculation of the new concentrations $C_{i}(t+\Delta t, j \cdot \Delta z)$ and $C_{p}(t+\Delta t, j \cdot \Delta z)$ after an irradiation time $\Delta t$ (eqs S13-S14); (iii) calculation of the diffusive transport for the interval $\Delta \mathrm{t}$; (iv) return to (i) and so on. For every numerical cycle the reflectance of the irradiated layer is calculated with eqs S7-S10 and the quantum yield is determined by least square minimization of the error between experimental and modeled values.

\section{Literature cited}

(1) Schwarzenbach, R. P.; Gschwend, P. M.; Imboden, D. M. Environmental organic chemistry; 2nd ed.; John Wiley and Sons: Hoboken, 2003.

(2) Ciani, A.; Goss, K.-U.; Schwarzenbach, R. P. Light penetration in soil and particulate minerals, Eur. J. Soil Sci. 2005, accepted.

(3) Ciani, A.; Goss, K.-U.; Schwarzenbach, R. P. Determination of Molar Absorption Coefficients of Organic Compounds Adsorbed in Porous Media, Chemosphere 2005, accepted. 
(4) Gade, R.; Porada, T. Quantitative determination of photokinetic parameters for reversible photoisomerizations in light scattering adsorbant-adsorbates, Z. Phys. Chem. 2000, 214, 149-167.

(5) Gade, R. A new method for determining quantum yields of photochemical reactions in powdered adsorbent-adsorbates, Z. Phys. Chem. 1995, 189, 99-111.

(6) Kubelka, P. New contributions to the optics of intensely light-scattering materials. Part I, J. Opt. Soc. Am. 1948, 38, 448-457 + errata on page 1067.

(7) Oelkrug, D.; Honnen, W.; Wilkinson, F.; Willsher, C. J. Modeling of transient production and decay following laser excitation of opaque materials, Journal of the Chemical Society Faraday Transactions II 1987, 83, 2081-2095.

(8) Moser-Boroumand, F. Spectroscopie FTIR quantitative de réflectance et transmittance diffuses, Ph.D. thesis, EPF Lausanne: Lausanne, 1991.

(9) Mammel, U.; Brun, M.; Oelkrug, D. Absorption and emission spectroscopy in scattering media: Modelling of fluorescence decay curves and photometric laws for systems with inhomogeneous absorber distribution, Fresenius' Journal of Analytical Chemistry 1992, 344, 147-152.

(10) Kubelka, P. New contributions to the optics of intensely light scattering materials. Part II: Nonhomogeneous layers, J. Opt. Soc. Am. 1954, 44, 330-335. 